\title{
Efecto de agentes blanqueadores libres de peróxido sobre el color dental. Revisión sistemática*
}

Effect of Peroxide-Free Dental Bleaching Agents on Dental Color. Systematic Review Fecha de recepción: 02-05-2018 I Fecha de aceptación: 05-12-2018

\section{INGRID GIOVANA SUÁREZ FAJARdo}

Universidad Nacional de Colombia. Bogotá, Colombia. igsuarezf@unal.edu.co. https://orcid.org/0000-0002-9475-6292

\begin{abstract}
MAURicio Rodríguez GodoY
Universidad Nacional de Colombia. Bogotá, Colombia. mrodriguezgo@unal.edu.co. https://orcid.org/0000-0002-5409-8264
\end{abstract}

\section{Edgar Delgado Mejía}

Universidad Nacional de Colombia. Bogotá, Colombia. edelgadom@unal.edu.co. https://orcid.org/0000-0001-8170-1279

\section{CAROLINA TORRES Rodríguez ${ }^{\mathrm{a}}$}

Universidad Nacional de Colombia. Bogotá, Colombia. ctorresr@unal.edu.co. https://orcid.org/0000-0003-1416-8472 
*Investigación original, revisión sistemática

a'Correspondencia: ctorresr@ unal.edu.co

doi: https://doi.org/10.11144/Javeriana.uo37-79.eabp

Cómo citar: Suárez Fajardo IG, Rodríguez Godoy M, Delgado Mejía E, Torres Rodríguez C. Efecto de agentes blanqueadores libres de peróxido sobre el color dental. Revisión sistemática. Univ Odontol. 2018 jul-dic; 37(79). doi: https://doi.org/10.11144/Javeriana.uo37-79.eabp

\section{RESUMEN}

Antecedentes: Los agentes blanqueadores oxidantes tales como los peróxidos generan daños irreversibles en el esmalte dental y afectan químicamente el componente orgánico e inorgánico del esmalte. Se reportan en la literatura sustancias alternativas que pueden mejorar el color del esmalte, sin causarle daño. Objetivo: Identificar las sustancias blanqueadoras tipo remineralizante reportadas en la literatura y su efecto en el color del esmalte dental. Métodos: Se consultaron las bases de datos PubMed, ScienceDirect, Embase, Scielo, Lilacs y Scopus, las palabras clave empleadas para la búsqueda fueron dental enamel, tooth bleaching, bleaching, calcium phosphate, hidroxyapatite, apatite, biomimetic, biomimetics, conectadas por el operador booleano AND y OR de diferentes maneras. Los criterios de elegibilidad de los artículos que harían parte de la revisión fueron que no incluyeran peróxidos de hidrógeno y carbamida con adición fluoruros y fosfatos de calcio y adicionalmente que emplearan un método de medición de color. Resultados: 
El resultado de la búsqueda arrojó 7 artículos, las sustancias encontradas de tipo remineralizante fueron hidroxiapatita sintética, fosfatos de calcio y el hexametafosfato de sodio. Según los criterios de evaluación definidos solo 4 de ellos tuvieron un nivel de evidencia alto, uno nivel medio y dos bajos. Todos los estudios reportan con los tratamientos probados, la capacidad de generar cambios en el color del esmalte dental. Conclusión: Las sustancias blanqueadoras remineralizantes encontradas, tienen la capacidad de producir cambios en el color del esmalte dental, lo cual se evidencia con modificación en las diferentes escalas de medición empleadas.

\section{Palabras clave}

biomiméticos; blanqueamiento dental; esmalte dental; fosfato de calcio; hidroxiapatita; materiales biomiméticos

\section{Áreas temáticas}

color; materiales dentales; odontología

\section{ABSTRACT}

Background: Oxidizing bleaching agents such as peroxides generate irreversible damage to dental enamel and chemically affect the organic and inorganic component of the enamel. Alternative substances that can improve the color of the enamel without damaging it are reported in the literature. Purpose: To identify the remineralizing bleaching substances reported in the literature and their effect on the color of the dental enamel. Methods: The databases PubMed, ScienceDirect, Embase, SciELO, Lilacs and Scopus were consulted, the keywords used for the search were dental 
enamel, tooth bleaching, bleaching, calcium phosphate, hydroxyapatite, apatite, biomimetic, biomimetics, connected by the Boolean operator AND and OR in different ways. The eligibility criteria of the articles that would be part of the review were not to include hydrogen peroxide and carbamide peroxides with addition of fluorides and calcium phosphates and additionally using a color measurement method. Results: The result of the search yielded 7 articles, the substances found of remineralizing type were synthetic hydroxyapatite, calcium phosphates and sodium hexametaphosphate. According to the evaluation criteria defined, only 4 of them had a high level of evidence, one medium level and two low. All studies report with proven treatments the ability to generate changes in tooth enamel color. Conclusions: The remineralizing whitening substances found have the ability to produce changes in the color of the dental enamel, which is evidenced with modification in the different measurement scales used.

\section{Keywords}

biomimetic materials; biomimetics; calcium phosphate; hydroxyapatite; tooth bleaching; tooth enamel

\section{Thematic fields}

color; dental materials; dentistry

\section{INTRODUCCIÓN}

Con la evolución de la odontología y los materiales dentales se han desarrollado técnicas con el fin de mejorar la estética dental y responder las demandas de los pacientes. Es este el caso del 
blanqueamiento dental que surge como una respuesta a la preocupación de los pacientes por modificar el color de sus dientes a tonos más "blancos".

Existen diversos métodos de blanqueamiento como los son mecánicos, ópticos, químicos, y remineralizantes (1). Los métodos mecánicos utilizan partículas abrasivas a base de carbonato de calcio, bicarbonato de sodio, sílice precipitada, piedra pómez y perlita las cuales remueven de la superficie dental manchas extrínsecas; sin embargo pueden llegar a alterar y afectar la superficie del esmalte por desgaste, sí su uso es excesivo (2). El azul de covarina es un material usado en método óptico que se deposita en la superficie dental $(3,4)$, generando cambios en el parámetro $b$ del color (Sistema CIELAB) (5-7). Dentro de los métodos químicos se encuentran el uso de los peróxidos de hidrógeno y carbamida, los cuales producen el efecto de cambio de color por medio de oxidación y son los más usados en la actualidad tanto en técnicas de consultorio como casero (8). Sin embargo estas sustancias oxidantes tan utilizadas en los pacientes por su efectividad en el cambio de color, son también citotóxicas, pueden generar daño pulpar, producen sensibilidad, generan desorganización estructural, pérdida de sustancia interprismática y cambios químicos en por pérdida de calcio y carbonatos (9-12). Actualmente los fabricantes de los materiales blanqueadores a base de peróxidos, les han agregado iones fosfato de calcio amorfo e iones de flúor en diferentes concentraciones con el fin de disminuir la pérdida de la dureza del esmalte y los posibles cambios morfológicos en el mismo. Se plantea que estos iones saturan la sustancia blanqueadora, lo cual permite un intercambio que repone el flúor y calcio perdidos. Teóricamente de esta manera el esmalte recupera su ácido resistencia $(13,14)$. Existe otro agente químico acomplejante-secuestrante, como el hexametafosfato de sodio que disminuye las pigmentaciones, 
incrementa el valor y disminuye los parámetros $\mathrm{a}^{*} \mathrm{y} \mathrm{b}^{*}$ del color pero tiene el efecto de disolver el esmalte.

Debido a los efectos que tienen los agentes blanqueadores sobre la superficie y estructura, se han reportado en la literatura el desarrollo de un cuarto grupo de agentes remineralizantes alternativos a los peróxidos, que también mejoran el color dental pero de manera segura. Sustancias como la hidroxiapatita, fosfatos de calcio, zinc carboapatita, fosfato tricálcico TCP, (1), según los estudios pueden inducir modificación en los parámetros CIELAB del color $\left(L^{*}, a^{*}\right.$ y b*) sin producir daños a la estructura dental (sustancias blanqueadoras remineralizantes) (1). La hidroxiapatita es capaz de modificar el brillo y aumentar el valor, así como el fosfato tricálcico y la zinc carbonoapatita modifican el delta E (parámetro que indica el cambio de color) (15) y aumentan el L*(valor). Sin embargo falta aún suficiente soporte científico, calidad de las mediciones del color y desarrollo, para la implementación de estas sustancias como agentes blanqueadores dentales en los humanos. Por eso se planteó que la pregunta de investigación para la presente revisión ¿Cuál es el efecto sobre el color del esmalte dental de agentes blanqueadores libres de peróxido? El objetivo de la presente revisión sistemática es identificar el efecto sobre el color dental de agentes blanqueadores libres de peróxido reportados en la literatura.

\section{MATERIALES Y MÉTODOS}

Inicialmente se consultó en las bases de datos Cochrane, Próspero y Tripdatase, Epistemónikos en las cuales no se encontraron revisiones sistemáticas, ni metanálisis publicados referentes a agentes blanqueadores libres de peróxido. Para la búsqueda se consultaron las bases de datos PubMed, 
ScienceDirect, Embase, Scielo, Lilacs y Scopus, así como también se realizó búsqueda manual. Las palabras clave empleadas para la búsqueda fueron dental enamel, tooth bleaching, bleaching, calcium phosphate, hidroxyapatite, apatite, biomimetic, biomimetics, conectadas por el operador booleano AND y OR de diferentes maneras. Se establecieron múltiples ecuaciones de búsqueda, ver tabla 1. Los límites para la búsqueda fueron año 2000 a 2016, idioma inglés, artículos completos o resúmenes que hayan estudiado sustancias blanqueadoras diferentes a los peróxidos y estudios experimentales. Los criterios de elegibilidad de los artículos que hicieron parte de la revisión fueron que no incluyeran peróxidos de hidrógeno y carbamida con adición fluoruros y fosfatos de calcio y adicionalmente que emplearan un método de medición de color.

La búsqueda arrojó 41 artículos que se seleccionaron por título, los demás se descartaron por tratar temas distintos al tópico de interés. Se leyeron los resúmenes de los 41 artículos, de los cuales se descartaron 35 debido a que incluían peróxidos de hidrógeno y carbamida con adición de fluoruros y fosfatos de calcio. Finalmente se obtuvieron 3 artículos con las ecuaciones de búsqueda utilizadas y 4 artículos encontrados por búsqueda manual, de los cuales uno fue un resumen. Los textos completos se adquirieron por medio del Sistema Nacional de Bibliotecas de la Universidad Nacional de Colombia (Bases de datos y revistas electrónicas disponibles) y uso del servicio de obtención de documentos de la Universidad Nacional de Colombia (figura 1). 
FIGURA 1

FLUJO GRAMA DE BÚSQUEDA Y SELECCIÓN DE ARTÍCULOS

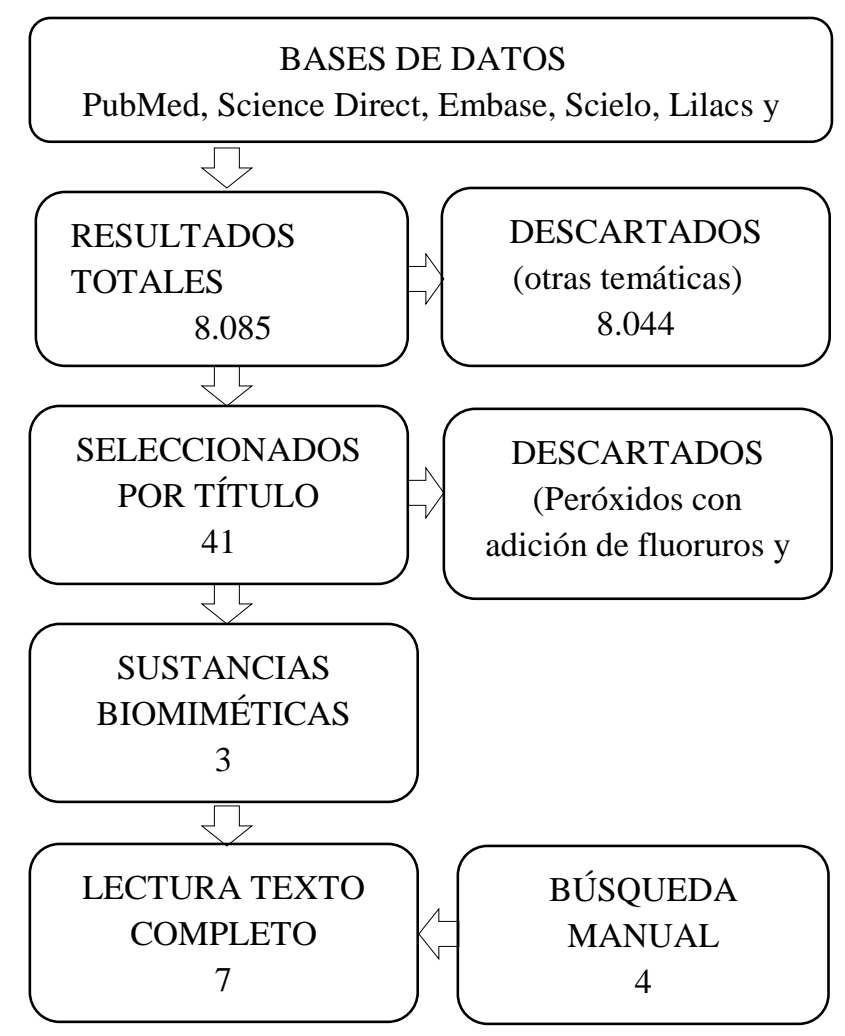

La evaluación de los artículos fue realizada únicamente por la autora principal del artículo. No se utilizó la herramienta de evaluación de riesgo de sesgos para estudios clínicos aleatorizados de la colaboración Cochrane, ni la herramienta New Castle Otawa para evaluar la calidad metodológica de estudios observacionales pues no aplicaban para los estudios incluidos en la revisión. Entonces, se utilizaron 5 criterios de evaluación de sesgos de los 7 artículos seleccionados: 1. Entrenamiento y nivelación de los operadores y calibración de tecnología utilizada en la medición del cambio de color (de estar reportado obtendrá 1 punto). 2. Técnica utilizada para evaluación del cambio de color (Técnicas objetivas: colorímetros, espectrofotómetros, obtendrán un punto). 3. Sistema de medición de color empleado (Sí se reporta uso de CIELAB, obtendrá un punto). 4. Existencia de un grupo control (Comparación con otras sustancias remineralizantes o un control sin tratamiento, en ese caso obtendrá un punto) 5. Existencia de análisis estadístico (Pruebas estadísticas que 
comparen el cambio de color entre los grupos evaluados, de estar reportado obtendrá un punto);

estos criterios se seleccionaron para dar información respecto a la rigurosidad como se realizaron

los estudios, sí se controlaron los sesgos y sí trabajan con los equipos y sistemas de medición

pertinentes. Se estableció una escala de 0 a 5 puntos, para medir nivel de evidencia, estableciéndose

alto con un puntaje entre 4 y 5 , medio con 3 puntos y bajo entre 1 y 2 puntos (tablas 1 y 2 ).

TABLA 1

ESTRATEGIA DE BÚSQUEDA DE LOS 7 ARTÍCULOS SELECCIONADOS

\begin{tabular}{|c|c|}
\hline Estrategia de búsqueda & Resultado \\
\hline $\begin{array}{l}\text { Búsqueda manual: Artículos encontrados } \\
\text { por estar referenciados en otros artículos o } \\
\text { documentos. }\end{array}$ & $\begin{array}{l}\text { - Guo C, Liu H, Katayama I. Effect of hydroxyapatite toothpaste } \\
\text { on vital tooth color. J Dent Res. 2002;8:A254 (20) } \\
\text { Niwa M, Sato T, Li W, Aoki H, Aoki H, Daisaku T. Polishing } \\
\text { and whitening properties of toothpaste containing } \\
\text { hydroxyapatite. J Mater Sci Mater Med. } 2001 \text { Mar;12(3):277- } \\
\text { 81. (21) } \\
\text { Vargas P. Delgado E. Torres E. Effect of a new remineralizing } \\
\text { biomaterial on the color of dental enamel. Acta Odontol. } \\
\text { Latinoam. 2014. Vol. } 27 \mathrm{~N}^{\circ} 1 \text { / 2014 / 3-8 (19) } \\
\text { Pentapati KC, Sabnis N, Shrikhande B, Kashyap R, } \\
\text { Kukkamalla MA. Stain Removal Efficacy of Herbal Mouth } \\
\text { Rinses Containing Sodium Hexametaphosphate. J Res Med } \\
\text { Educ Ethics. 2014;4(2):165-70. (18) }\end{array}$ \\
\hline $\begin{array}{l}\text { Pubmed } \\
\text { - } \quad \text { calcium phosphate AND tooth } \\
\text { bleaching } \\
\text { - } \quad \text { hydroxyapatite AND tooth bleaching } \\
\text { - } \quad \text { hydroxyapatite AND bleaching } \\
\text { calcium phosphate AND tooth } \\
\text { bleaching AND hydroxyapatite } \\
\text { - calcium phosphate AND bleaching } \\
\text { - } \quad \text { apatite AND bleaching } \\
\text { spectrophotometry AND tooth } \\
\text { whitening" }\end{array}$ & $\begin{array}{l}\text { Jin J, Xu X, Lai G, Kunzelmann K-H. Efficacy of tooth } \\
\text { whitening with different calcium phosphate-based } \\
\text { formulations. Eur J Oral Sci. } 1 \text { de agosto de 2013;121(4):382- } \\
\text { 8. (17) }\end{array}$ \\
\hline $\begin{array}{l}\text { Pubmed } \\
\text { - calcium phosphate AND tooth } \\
\text { bleaching } \\
\text { - calcium phosphate AND bleaching }\end{array}$ & $\begin{array}{l}\text { - Gerlach RW, Ramsey LL, Baker RA, White DJ. Extrinsic stain } \\
\text { prevention with a combination dentifrice containing calcium } \\
\text { phosphate surface active builders compared to two marketed } \\
\text { controls. J Clin Dent. 2002;13(1):15-8. (22) }\end{array}$ \\
\hline $\begin{array}{l}\text { Pubmed } \\
\text { - } \quad \text { hydroxyapatite AND tooth bleaching } \\
\text { - } \quad \text { hydroxyapatite AND bleaching } \\
\text { - } \quad \text { biomimetic AND bleaching AND } \\
\quad \text { dental enamel } \\
\text { - } \quad \text { apatite AND bleaching }\end{array}$ & $\begin{array}{l}\text { Dabanoglu A, Wood C, García-Godoy F, Kunzelmann K-H. } \\
\text { Whitening effect and morphological evaluation of } \\
\text { hydroxyapatite materials. Am J Dent. febrero de } \\
\text { 2009;22(1):23-9. (16) }\end{array}$ \\
\hline $\begin{array}{l}\text { Embase } \\
\text { - } \quad \text { hydroxyapatite AND tooth bleaching } \\
\text { - } \quad \text { hydroxyapatite AND bleaching }\end{array}$ & \\
\hline
\end{tabular}


TABLA 2

\begin{tabular}{|c|c|c|c|c|c|c|c|c|}
\hline Referencia & $\begin{array}{l}\text { Sustancia } \\
\text { evaluada }\end{array}$ & $\begin{array}{l}\text { 1. Entrenamiento } \\
\text { y nivelación de los } \\
\text { operadores y } \\
\text { calibración de } \\
\text { tecnología } \\
\text { utilizada (1 punto) }\end{array}$ & $\begin{array}{l}\text { 2. Técnica } \\
\text { utilizada para } \\
\text { evaluación del } \\
\text { cambio de color } \\
\text { (1 punto) }\end{array}$ & $\begin{array}{l}\text { S ANALIZADOS } \\
\text { 3.Sistema } \\
\text { de medición } \\
\text { de color (1 } \\
\text { punto) }\end{array}$ & $\begin{array}{l}\text { 4.Grupo } \\
\text { control }(1 \\
\text { punto) }\end{array}$ & $\begin{array}{l}\text { 5.Análisis } \\
\text { estadístico (1 } \\
\text { punto) }\end{array}$ & Resultados & $\begin{array}{l}\text { Nivel de } \\
\text { Evidencia }\end{array}$ \\
\hline $\begin{array}{l}\text { Dabanoglu, } \\
2009 \text { (16) }\end{array}$ & $\begin{array}{l}\text { Nano- } \\
\text { hidroxiapatita, } \\
\text { nano- } \\
\text { hidroxiapatita } \\
\text { cristalina, fosfato } \\
\text { tricálcico en polvo } \\
\text { y nano- } \\
\text { hidroxiapatita } \\
\text { integrada a una } \\
\text { película } \\
\text { polimérica. }\end{array}$ & $\begin{array}{l}\text { Tres mediciones } \\
\text { promediadas. } \\
\text { Equipo calibrado } \\
\text { con bloque de } \\
\text { cerámica. } \\
\text { Uso de caja negra } \\
\text { para estandarizar } \\
\text { condiciones de luz }\end{array}$ & $\begin{array}{l}\text { Técnica objetiva. } \\
\text { Espectrofotómetr } \\
\text { o Vita } \\
\text { EasyShade. }\end{array}$ & CIELAB & $\begin{array}{l}\text { Se comparan } 4 \\
\text { presentaciones } \\
\text { de } \\
\text { hidroxiapatita } \\
\text { pero sin } \\
\text { control + o - }\end{array}$ & $\begin{array}{l}\text { ANOVA y } \\
\text { test de Tukey } \\
\text { post hoc }\end{array}$ & $\begin{array}{l}\text { Todas las } \\
\text { presentaciones } \\
\text { produjeron cambio } \\
\text { en el parámetro } \\
\text { delta E y en la } \\
\text { microscopia capa } \\
\text { de hidroxiapatita } \\
\text { adherida al } \\
\text { esmalte. }\end{array}$ & $\begin{array}{l}4 \text { puntos } \\
\text { Alto }\end{array}$ \\
\hline $\begin{array}{l}\text { Vargas, } 2014 \\
\text { (19) }\end{array}$ & $\begin{array}{l}\text { Sustancia } \\
\text { remineralizante } \\
\text { experimental a } \\
\text { base de fosfatos } \\
\text { de calcio }\end{array}$ & $\begin{array}{l}\text { No mencionan } \\
\text { entrenamiento de } \\
\text { operador. } \\
\text { Equipo calibrado } \\
\text { con bloque de } \\
\text { cerámica. }\end{array}$ & $\begin{array}{l}\text { Técnica objetiva. } \\
\text { Espectrofotómetr } \\
\text { o Vita } \\
\text { EasyShade. }\end{array}$ & CIELAB & $\begin{array}{l}\text { Dos grupos: } \\
\text { saliva } \\
\text { hipotónica y } \\
\text { saliva } \\
\text { isotónica }\end{array}$ & $\begin{array}{l}\text { ANOVA y } \\
\text { análisis de } \\
\text { varianza } \\
\text { no } \\
\text { paramétrico } \\
\text { Kruskal } \\
\text { Wallis }\end{array}$ & $\begin{array}{l}\text { La sustancia } \\
\text { remineralizante } \\
\text { afectó las variables } \\
\Delta \mathrm{b}, \Delta \mathrm{E} \text { y } \Delta \mathrm{L} .\end{array}$ & $\begin{array}{l}4 \text { puntos } \\
\text { Alto }\end{array}$ \\
\hline Jin, 2013 (17) & $\begin{array}{l}\text { Suspensión de } \\
\text { carboapatita de } \\
\text { zinc, suspensión } \\
\text { de fosfato } \\
\text { tricálcico, zinc- } \\
\text { carboapatita }\end{array}$ & $\begin{array}{l}\text { No mencionan } \\
\text { entrenamiento de } \\
\text { operador. } \\
\text { Equipo calibrado } \\
\text { con bloque de } \\
\text { cerámica. } \\
\text { Uso de caja negra } \\
\text { para estandarizar } \\
\text { condiciones de luz. }\end{array}$ & $\begin{array}{l}\text { Técnica objetiva. } \\
\text { Espectrofotómetr } \\
\text { o Vita } \\
\text { EasyShade. }\end{array}$ & CIELAB & $\begin{array}{l}\text { Controles: } \\
\text { Pasta dental } \\
\text { comercial y } \\
\text { flúor tópico }\end{array}$ & $\begin{array}{l}\text { ANOVA y } \\
\text { test de Tukey } \\
\text { post hoc } \\
\text { corregido con } \\
\text { Bonferroni }\end{array}$ & $\begin{array}{l}\text { Suspensión de } \\
\text { fosfato tricálcico al } \\
10 \% \text { produjo } \\
\text { cambio más } \\
\text { significativo de } \\
\text { color. Los } \\
\text { diferentes fosfatos } \\
\text { de calcio se } \\
\text { adhirieron al } \\
\text { esmalte }\end{array}$ & $\begin{array}{l}4 \text { puntos } \\
\text { Alto }\end{array}$ \\
\hline
\end{tabular}




\begin{tabular}{|c|c|c|c|c|c|c|c|c|}
\hline $\begin{array}{l}\text { Pentapati, } \\
2014 \text { (18) }\end{array}$ & $\begin{array}{l}\text { Enjuague bucal } \\
\text { con contenido de } \\
\text { hexametafosfato } \\
\text { de sodio, extracto } \\
\text { de aloe, clavo, } \\
\text { mentol y otros } \\
\text { aditivos. }\end{array}$ & $\begin{array}{l}\text { No reportan } \\
\text { entrenamiento o } \\
\text { nivelación de los } \\
\text { operadores } \\
\text { No mencionan } \\
\text { calibración del } \\
\text { equipo }\end{array}$ & $\begin{array}{l}\text { Técnica objetiva. } \\
\text { Cámara digital } \\
\text { de alta } \\
\text { resolución } \\
\text { Nikkon® Japan, } \\
\text { D300 y } \\
\text { analizadas con } \\
\text { Adobe } \\
\text { Photoshop } \\
\text { versión } 8.0\end{array}$ & CIELAB & $\begin{array}{l}\text { Comparado } \\
\text { con Listerine } \\
\text { Whitening }\end{array}$ & $\begin{array}{l}\text { ANOVA y } \\
\text { test de Tukey } \\
\text { post hoc }\end{array}$ & $\begin{array}{l}\text { Incremento } \\
\text { significativo en el } \\
\text { valor de los dientes } \\
\text { y una disminución } \\
\text { en el valor de los } \\
\text { parámetros a y b } \\
\text { del color. }\end{array}$ & $\begin{array}{l}4 \text { puntos } \\
\text { Alto }\end{array}$ \\
\hline $\begin{array}{l}\text { Guo, } 2002 \\
(20)\end{array}$ & $\begin{array}{l}\text { Hidroxiapatita } \\
\text { sintética }\end{array}$ & $\begin{array}{l}\text { No reportan } \\
\text { entrenamiento de } \\
\text { los operadores } \\
\text { No mencionan } \\
\text { calibración del } \\
\text { equipo }\end{array}$ & $\begin{array}{l}\text { Técnica objetiva. } \\
\text { Fotocolorímetro } \\
\text { Photoresearch } \\
\text { Spectra-Scan } \\
\text { PR-650. } \\
\text { Espectrofotómetr } \\
\text { o Murakami } \\
\text { CMS-35FS }\end{array}$ & CIELAB & $\begin{array}{l}\text { Control - } \\
\text { Grupo tratado } \\
\text { con crema sin } \\
\text { hidroxiapatita }\end{array}$ & No tiene & $\begin{array}{l}\text { Incremento en el L } \\
\text { y hubo variación en } \\
\text { el delta E, se formó } \\
\text { una capa adherida } \\
\text { al esmalte. }\end{array}$ & $\begin{array}{l}3 \text { puntos } \\
\text { Medio }\end{array}$ \\
\hline $\begin{array}{l}\text { Gerlach, } \\
2002(22)\end{array}$ & $\begin{array}{l}\text { Crema dental con } \\
\text { hexametofosfato y } \\
\text { pirofosfato } \\
\text { soluble }\end{array}$ & $\begin{array}{l}\text { No menciona } \\
\text { entrenamiento o } \\
\text { nivelación de los } \\
\text { examinadores }\end{array}$ & $\begin{array}{l}\text { Técnica visual } \\
\text { subjetiva }\end{array}$ & $\begin{array}{l}\text { Test de } \\
\text { Lobene }\end{array}$ & $\begin{array}{l}\text { Control sin } \\
\text { tratamiento } \\
\text { Control }+ \\
\text { pasta dental } \\
\text { abrasiva } \\
\text { comercial }\end{array}$ & $\begin{array}{l}\text { Análisis de } \\
\text { covarianza } \\
\text { (ANCOVA) }\end{array}$ & $\begin{array}{l}\text { Disminución en la } \\
\text { cantidad de } \\
\text { pigmentaciones en } \\
\text { un } 33 \% \text { en el grupo } \\
\text { tratado con la } \\
\text { crema experimental }\end{array}$ & $\begin{array}{l}2 \text { puntos } \\
\text { Bajo }\end{array}$ \\
\hline $\begin{array}{l}\text { Niwa, } 2001 \\
\text { (21) }\end{array}$ & $\begin{array}{l}\text { Crema dental con } \\
\text { hidroxiapatita al } \\
3 \% \text { y } 15 \%\end{array}$ & $\begin{array}{l}\text { No reportan } \\
\text { entrenamiento de } \\
\text { los operadores } \\
\text { No mencionan } \\
\text { calibración del } \\
\text { equipo }\end{array}$ & $\begin{array}{l}\text { Técnica objetiva. } \\
\text { Colorímetro SZ- } \\
\text { Y-90 y SE-200. }\end{array}$ & $\begin{array}{l}\text { Sistema de } \\
\text { Kobayashi } \\
\text { para } \\
\text { determinació } \\
\text { n de } \\
\text { blanqueamie } \\
\text { nto y brillo }\end{array}$ & $\begin{array}{l}\text { Control } \\
\text { positivo: } \\
\text { Grupo tratado } \\
\text { con crema sin } \\
\text { hidroxiapatita }\end{array}$ & No tiene & $\begin{array}{l}\text { Aumento en el } \\
\text { brillo y "blancura", } \\
\text { siendo mayor con } \\
\text { la concentración de } \\
15 \%\end{array}$ & $\begin{array}{l}2 \text { puntos } \\
\text { Bajo }\end{array}$ \\
\hline
\end{tabular}




\section{RESULTADOS}

El resultado de la búsqueda arrojó 7 artículos (tabla 2). Los agentes evaluados fueron la hidroxiapatita sintética en diferentes presentaciones (cristalina, integrada a una película polimérica, etc.) y concentraciones (3\% y $15 \%)$, sustancias basadas en diferentes fosfatos de calcio y el hexametafosfato de sodio combinados con pirosfosfatos.

Según los criterios de evaluación definidos, solo 4 de los 7 artículos tuvieron un nivel de evidencia alto [Dabanoglu A 2009 (16), Jin J 2013 (17), Pentapati KC 2014 (18), Vargas PK 2014 (19)], ya que cumplieron cuatro de los cinco criterios establecidos. Un artículo tuvo nivel de evidencia medio [Guo C 2002 (20)], ya que empleó una técnica de medición del color objetiva, expresó sus resultados en Cie Lab y evaluó un grupo control. Dos artículos tuvieron nivel de evidencia bajo [Niwa M 2001 (21), Gerlach R 2002 (22)], debido a que no trabajaron con sistema Cie Lab, no reportan calibración de equipos, ni nivelación de observadores, uno de ellos empleó técnica visual para medición del color y el otro no realizó comparación estadística.

Cinco de los siete artículos presentaron en su desarrollo un análisis estadístico, lo cual indica que desde ese punto de vista hay un análisis objetivo de los datos, que aumenta la veracidad de las comparaciones. Guo C 2002 (20) y Niwa M 2001 (21) no reportaron análisis estadístico. Los estudios de Dabanoglu A, 2009. (16) y Vargas 2014 (19) no tuvieron en cuenta un grupo control en su metodología. 
Los artículos no mencionaron el entrenamiento y nivelación del operador que realizó las mediciones de color, lo cual es una desventaja metodológica, solamente Dabanoglu A, 2009, realizó tres mediciones a cada espécimen, que fueron promediadas.

Respecto a la tecnología utilizada y calibración de los equipos, todos los artículos realizaron medición del color con métodos objetivos, como son el uso de espectrofotómetro y colorímetro. Solamente el estudio de Gerlach R 2002, reportó medición subjetiva, ya que el análisis fue visual, lo cual dificulta que este artículo sea comparable con los demás y en general con toda la literatura disponible.

De los 7 artículos, 5 expresaron sus resultados de medición de color en la escala CIELAB, lo cual es un parámetro muy importante para dar validez a los mismos. Dada la heterogeneidad en la metodología y la presentación de los resultados de los artículos seleccionados, no se llevó acabo un metanálisis.

\section{DISCUSIÓN}

Teniendo en cuenta que el problema planteado fue los efectos deletéreos que tienen los agentes blanqueadores de tipo químico, se formuló la pregunta de investigación ¿Cuál es el efecto sobre el color del esmalte dental de agentes blanqueadores libres de peróxido? Para responder la pregunta se hizo una revisión sistemática de la literatura de 7 artículos seleccionados y se determinó que todas las sustancias remineralizantes reportadas en la literatura evaluadas (hidroxiapatita sintética, fosfato tricálcico, zinc-carboapatita, fosfatos de calcio y hexametafosfato de sodio), lograron 
producir modificaciones en el color, lo cual se estableció cuantitativamente por medio de la escala CIELAB y las otras escalas manejadas.

La hidroxiapatita sintética es una de las sustancias que se han planteado como alternativa para blanqueamiento dental por si sola. Es el principal componente inorgánico de los huesos y también se encuentra en el esmalte y dentina; es biocompatible, osteoconductiva, estable químicamente y no es tóxica. Se ha utilizado en el reemplazo de zonas de hueso, recubrimiento de implantes, liberador de medicamentos, entre otros (23). La hidroxiapatita se ha agregado a cremas dentales con el fin de prevenir la caries, tratar la enfermedad periodontal, disminuir la halitosis por medio de la absorción de proteínas, lípidos, polisacáridos y otras moléculas (24). Niwa y Sato en el 2001, evaluaron una crema dental con hidroxiapatita, la cual fue usada por pacientes a concentraciones de $3 \%$ y $15 \%$. Se realizaron mediciones con un colorímetro antes del tratamiento, a las dos semanas, un mes y seis meses de uso. Como resultado encontraron un aumento en el parámetro $L^{*}$ de los dientes tratados, siendo mayor con la concentración de $15 \%$. Los autores explican que se logra el efecto de blanqueamiento, porque la hidroxiapatita remineraliza el esmalte y esto hace que el este sea más liso, de este modo una superficie lisa aumenta el índice de reflexión de la luz (21).

Se ha evaluado también in vitro el efecto de la nano-hidroxiapatita, nano-hidroxiapatita cristalina, fosfato tricálcico en polvo y nano-hidroxiapatita integrada a una película polimérica, sobre el color dental. En este estudio se realizaron las mediciones del color con un espectrofotómetro Vita Easy Shade, pretratamiento y postratamiento cada 24 horas por 4 días y se observaron con microscopia electrónica de barrido. Se encontró que todas las formas probadas de hidroxiapatita produjeron un cambio estadísticamente significativo en el parámetro delta $\mathrm{E}$ y se formó una capa delgada de 
hidroxiapatita que reacciona con la hidroxiapatita natural del diente, por su proximidad química. (16).

En el estudio de Guo del 2002 probaron el efecto de una crema dental con hidroxiapatita sobre el color dental en voluntarios durante un mes, realizando mediciones con un fotocolorímetro cada tercer día. También se aplicó la crema a dientes extraídos, para luego ser observados con microscopia electrónica. Se encontró incremento en el parámetro L, variación en el delta E y se evidenció en los dientes extraídos que la hidroxiapatita formó una capa adherida al esmalte (20).

Diferentes fosfatos de calcio también han sido probados como agentes blanqueadores, mostrando resultados satisfactorios. Jin, et al, en el 2013, evaluaron el efecto in vitro en el color dental de una suspensión de zinc-carboapatita, suspensión de fosfato tricálcico y como control una crema blanqueadora abrasiva existente en el mercado. Como parámetro del cambio de color se empleó el delta E, medido con un espectrofotómetro Vita Easy Shade pretratamiento y cada 24 horas luego de la aplicación de las sustancias durante 4 días. Encontraron que la suspensión de fosfato tricálcico al $10 \%$, produjo el mayor cambio en delta E y la zinc-carbonato-apatita al $20 \%$ mostró los menores valores en delta E. Los diferentes fosfatos de calcio se adhirieron al esmalte. Mencionan los autores como posible causa del cambio de color, la formación de una capa superficial, que favorece la reflexión difusa, siendo más efectivas las sustancias probadas que tenían menor tamaño de partícula (17).

Vargas et al. en 2014 plantearon una sustancia remineralizante basada en compuestos de calcio y fosfato. Se evaluó su efecto sobre el color en 104 dientes humanos extraídos. Se calcularon los 
parámetros de color y los índices de blanqueamiento W, WIO y WIC pre y postratamiento con un espectrofotómetro Vita Easy Shade®. Como resultado encontraron que la sustancia modificó las variables $\mathrm{a}^{*}, \mathrm{~b}^{*}, \mathrm{E}$ y el índice de blanqueamiento WIO. (19)

Existen también reportes en la literatura del uso del pirofosfato soluble, como sustancia empleada para el control de las manchas. El pirofosfato soluble como el sódico es un secuestrante poderoso y retiene los iones calcio en la saliva y en agua y no en el esmalte, lo que produce disolución del esmalte y blanqueamiento. En el estudio de Gerlach y col del 2002 se evaluó el uso de una crema dental con contenido de hexametofosfato y pirofosfato soluble, alternada con el uso de crema de clorhexidina y té como agentes pigmentantes en pacientes. Se midió la presencia de manchas con el test de Lobene, pretratamiento, a las tres y seis semanas. Encontraron disminución en la cantidad de pigmentaciones en un 33\% en el grupo tratado con la crema experimental, con respecto al grupo control. (22).

Otra sustancia empleada para remover manchas ha sido el hexametafosfato de sodio combinado con pirofosfato, del cual se reporta efectividad para tratar pigmentaciones extrínsecas. Se ha utilizado en presentación de crema y enjuague bucal. En el trabajo de Pentapati en el 2014, se evaluó en dientes extraídos un enjuague bucal con contenido de hexametafosfato de sodio, extracto de aloe, clavo, mentol y otros aditivos. Los dientes fueron sumergidos en una solución de té y previo a esto se grabaron con ácido fosfórico al 37 \%, para favorecer la formación de manchas. Posteriormente se trataron los dientes con el enjuague experimental durante un minuto por 14 días. El cambio de color se evaluó tomando fotografías a los especímenes pre y postratamiento y analizándolas con el programa Adobe Photoshop versión 8.0, el cual trabaja con sistema CIELAB. 
Como resultado se encontró incremento significativo en el parámetro $L^{*}$ de los dientes a los 14 días y una disminución en el valor de los parámetros $a^{*}$ y b* del color. Los anteriores resultados según los autores, se basan en que el hexametafosfato de sodio tiene una fuerte atracción a la hidroxiapatita de calcio, adicionalmente su capacidad para romper las películas de las manchas extrínsecas y su retención en la superficie del diente para prevenir la nueva aparición de manchas (18).

Los anteriores estudios mencionados demuestran que la tendencia actual en la investigación de materiales dentales apunta a tomar la naturaleza y sus mecanismos como fuente de inspiración, desarrollando materiales más biocompatibles, seguros y efectivos. Sin embargo, se encuentra que no todos presentan un nivel de evidencia alto, que permita tomarlos como referente en el tema.

El método de medición de color en los artículos estudiados varía, utilizando algunas técnicas subjetivas visuales, cámaras digitales, colorímetros y espectrofotómetros; así mismo se manejan diferentes sistemas de medición del color, desde los más elaborados como el CIELAB, hasta otros menos objetivos como el test de Kobayashi y Lobene. Esta variabilidad entre los estudios impide que sean comparables entre sí y poder sacar conclusiones confiables de esta búsqueda sistemática; así mismo ocurre con el hecho que no todos emplean un método estadístico que establezca números de muestra mínimos que permitan el procesamiento de los resultados e identifiquen la significancia de las diferencias encontradas. Sin embargo, a pesar de las limitaciones mencionadas, todos los estudios reportan con los tratamientos probados, la capacidad de generar cambios en el color del esmalte dental. Se describen cambios en los parámetros CIELAB del color $L^{*}, a^{*}, b^{*}$ y $\Delta E$, aumento en la "blancura" y brillo, disminución en el número de manchas y en algunos casos 
adherencia a la superficie del esmalte de las sustancias probadas. Lo cual responde a la pregunta de investigación de la presente revisión.

La poca literatura encontrada del tema es una limitación de la presente revisión, sin embargo también es una fortaleza ya que no se encontró en la búsqueda revisiones sistemáticas publicadas de agentes blanqueadores libres de peróxido. Por esta razón es pertinente la realización del presente escrito y futuras investigaciones experimentales y clínicas en el tema, sistemas de medición subjetivos y objetivos CIELAB 2000, los índices y todos los procesos para poder llegar a uso humano.

\section{CONCLUSIONES}

Agentes blanqueadores libres de peróxido como hidroxiapatita sintética, fosfato tricálcico, zinccarboapatita, fosfatos de calcio y hexametafosfato de sodio tienen la capacidad de producir cambios en el color del esmalte dental según las escalas de medición del color empleadas.

Todos los estudios que se basaron en la escala CIELAB para medición del color, reportaron

cambios en el parámetro delta E. Algunos reportan cambios en $\mathrm{L}^{*} \mathrm{y}$ otros en los parámetros a* y $b^{*}$.

\section{RECOMENDACIONES}


A pesar que existe la tendencia a investigar sobre sustancias no oxidantes que modifiquen el color dental, aún falta desarrollo al respecto, que permita tener estas opciones disponibles para uso clínico.

\section{AGRADECIMIENTOS}

Universidad Nacional de Colombia, Vicerrectoría de Investigación, Maestría en Odontología, Centro de Investigación y Extensión Facultad de Odontología. Proyecto registrado en Hermes código 26964. Aprobación Comité de Ética Facultad de Odontología No CIE 327-14 del 22 de enero de 2015.

\section{REFERENCIAS}

1. Pulido K. Sustancias blanqueadoras alternativas: Una revisión bibliográfica. Univ Nac Colomb. 2013;

2. Lewis R, Barber SC, Dwyer-Joyce RS. Particle motion and stain removal during simulated abrasive tooth cleaning. 16th Int Conf Wear Mater. 10 de septiembre de 2007; 263(1-6): 18897.

3. Joiner A, Philpotts CJ, Alonso C, Ashcroft AT, Sygrove NJ. A novel optical approach to achieving tooth whitening. J Dent. 2008; 36, Suppl 1:S8-14

4. Joiner A. Whitening toothpastes: a review of the literature. J Dent. 2010;38 Suppl 2:e17-24.

5. Chu SJ, Trushkowsky RD, Paravina RD. Dental color matching instruments and systems. Review of clinical and research aspects. J Dent. 2010;38 Suppl 2:e2-16. 
6. Sikri VK. Color: Implications in dentistry. J Conserv Dent JCD. octubre de 2010;13(4): 24955.

7. Bersezio C, Oliveira Jr OB, Docencia L, Angel P, Estay J, Corral C. Instrumentación para el registro del color en odontología. Rev Dent Chile. 2014; 105(1): 8-12.

8. Dahl JE, Pallesen U. Tooth bleaching--a critical review of the biological aspects. Crit Rev Oral Biol Med Off Publ Am Assoc Oral Biol. 2003; 14(4): 292-304.

9. Morozov IA, Belyaev AY, Izyumov RI, Erofeeva ES, Gileva OS. Impact of whitening on the microstructure of human tooth enamel. Inorg Mater Appl Res. 2013; 4(1): 71-76.

10. Ogiwara M, Miake Y, Yanagisawa T. Changes in dental enamel crystals by bleaching. J Hard Tissue Biol. 2008;17(1):11-16.

11. Joiner A. Review of the effects of peroxide on enamel and dentine properties. J Dent. diciembre de 2007; 35(12): 889-96.

12. Rodríguez CT. Efectos microquímicos del peróxido de hidrógeno de alta concentración y el ácido fosfórico sobre la capa superficial y subsuperficial del esmalte bovino. Granada, España: Editorial de la Universidad de Granada; 2010.

13. Alexandrino L, Gomes Y, Alves E, Costi H, Rogez H, Silva C. Effects of a bleaching agent with calcium on bovine enamel. Eur J Dent. Julio de 2014; 8(3): 320-5.

14. Borges AB, Guimaräes CA, Bresciani E, Ramos CJ, Borges ALS, Rocha Gomes Torres C. Effect of incorporation of remineralizing agents into bleaching gels on the microhardness of bovine enamel in situ. J Contemp Dent Pract. abril de 2014; 15(2): 195-201.

15. Pecho OE, Ghinea R, Alessandretti R, Pérez MM, Della Bona A. Visual and instrumental shade matching using CIELAB and CIEDE2000 color difference formulas. Dent Mater. enero de 2016; 32(1): 82-92. 
16. Dabanoglu A, Wood C, García-Godoy F, Kunzelmann K-H. Whitening effect and morphological evaluation of hydroxyapatite materials. Am J Dent. febrero de 2009; 22(1): 239.

17. Jin J, Xu X, Lai G, Kunzelmann K-H. Efficacy of tooth whitening with different calcium phosphate-based formulations. Eur J Oral Sci. 1 de agosto de 2013; 121(4): 382-8.

18. Pentapati KC, Sabnis N, Shrikhande B, Kashyap R, Kukkamalla MA. Stain Removal Efficacy of Herbal Mouth Rinses Containing Sodium Hexametaphosphate. J Res Med Educ Ethics. $2014 ; 4(2): 165-70$.

19. Vargas-Sánchez PK, Delgado-Mejía E, Torres-Rodríguez C. Effect of a new remineralizing biomaterial on the color of dental enamel. Acta Odontol Latinoam. 2014; 27(1):3-8.

20. Guo C, Liu H, Katayama I. Effect of hydroxyapatite toothpaste on vital tooth color. J Dent Res. 2002; 81: A254-1964, San Diego Abstracts.

21. Niwa M, Sato T, Li W, Aoki H, Aoki H, Daisaku T. Polishing and whitening properties of toothpaste containing hydroxyapatite. J Mater Sci Mater Med. marzo de 2001; 12(3): 277-81.

22. Gerlach RW, Ramsey LL, Baker RA, White DJ. Extrinsic stain prevention with a combination dentifrice containing calcium phosphate surface active builders compared to two marketed controls. J Clin Dent. 2002; 13(1): 15-8.

23. Londoño M. Características cristaloquímicas de la hidroxiapatita sintética tratada a diferentes temperaturas. Rev EIA. junio de 2006;5: 109-18.

24. Dorozhkin SV. Amorphous calcium (ortho)phosphates. Acta Biomater. diciembre de 2010; 6(12): 4457-75. 